\title{
Performanceanalyse von zwei optimierten Messverfahren in der kapazitiven Füllstandmessung
}

\author{
Jens Matheis, Andreas Uhl, Dr. Thomas Föhn, Furkan Dayi \\ EBE Elektro-Bau-Elemente GmbH, Sielmingerstr. 63, 70771 Leinfelden-Echterdingen
}

\section{Zusammenfassung}

Die kapazitive Füllstandmessung ist ein etabliertes Verfahren zur Erfassung von Füllständen von Flüssigkeiten sowie Schüttgütern. Eine Erweiterung der Messmethodik mittels eines breitbandigen Rauschsignals wird in diesen Artikel einer Performance Analyse unterzogen und dem klassischen Charge-Transfer-Messverfahren gegenübergestellt. Das Anwendungsszenario ist ein außen angebrachter kapazitiver Sensor an einer Gerätewand, hinter derer das eigentliche Mediengefäß wechselbar positioniert ist. Die Präsenzerkennung des Mediengefäßes sowie dessen Füllstands ist Ziel des Versuchsaufbaus auf Basis diskreter Bauelemente. Im Anschluss erfolgte eine Modellierung der Sensormessstrecke mit einem RLC-Ersatzschaltbild im direkten Vergleich der gemessenen Werte. Hier zeigte sich, dass die Leitfähigkeit $\sigma_{M}$ des Mediums einen wesentlichen Einfluss bereits bei sehr dünnen Filmen generiert. Um eine sichere Präsenzerkennung des Mediengefäßes trotz Filmbildung zu gewährleisten, wurden elektrostatische Feldsimulationen durchgeführt, um die Kapazitätsänderung $\Delta \mathrm{C}$

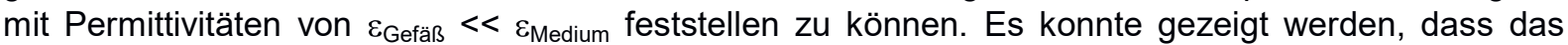
klassische Messverfahren auf Basis der Charge-Transfer-Technik für wässrige, leitfähige Medien die Anforderungen erfüllt. Bei viskosen, zähflüssigen Medien wie z.B. Öl oder Milch mit Neigung zur starken adhäsiven Filmbildung ist dagegen nur eine zuverlässigere Erfassung der Füllstände und der Mediengefäßpräsenz mit dem vorgestellten, rauschbasierten Verfahren möglich.

Keywords: kapazitive Füllstandmessung, kapazitive Füllstandschalter, adhäsive Filmbildung

\section{Einleitung}

Die Erfassung von Füllständen von Flüssigkeiten und Schüttgüter in der Industrie sind wohl verstanden und in den Märkten etabliert, z.B. als Schwellwertschalter. Zunehmend erhöht sich die Relevanz intelligenter Sensoren zur Füllstandmessung auch in dem Marktsegment der Medizintechnik sowie in den Produkten aus der Consumer-ElectronicsIndustrie.

Letztere Branche, welche sehr kostengerieben arbeitet, stellt erhöhte Anforderungen an Lösungen ohne den Einsatz von teuren Spezialbauelemente. Des Weiteren wird zunehmend gefordert, dass Sensoren für die Füllstanderkennung von wässrigen Lösungen auch die Präsenz von entnehmbaren Mediengefäßen erfassen und detektieren können mittels des gleichen Messprinzips.

Ein kapazitiv messender Sensor für wässrige, leitfähige Medien kann unsichtbar hinter einer oder mehreren dielektrischen Wänden verbaut werden (siehe Abbildung 1). Gegenüber konventionellen Reed-Schaltern bietet der kapazitive Sensor den Vorteil einer einfacheren Integration in das Gesamtsystem, ohne z.B. die Notwendigkeit einen Schwimmer zu verbauen, wodurch ein direkter Medienkontakt vermieden wird und Ausfällen durch Verkalkung und Verschmutzung und daraus folgendem Festsitzen der Schwimmer ausgeschlossen werden.

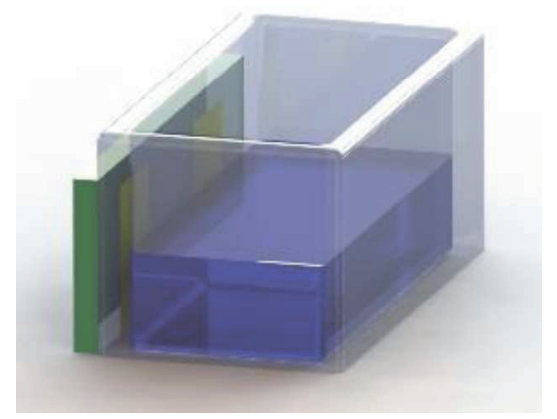

Abb. 1: Typische Aufgabenstellung einer kapazitiven Füllstandmessung inkl. Präsenzerkennung des Mediengefäßes.

Eine weitere Verbesserung ist die Möglichkeit zur kontinuierlichen Erfassung des tatsächlichen Füllstandes anstatt eines reinen Schwellwertschalters. 
Dabei stellen insbesondere elektrisch leitfähige Flüssigkeiten besondere Herausforderungen an die kapazitive Messtechnik, da bereits dünne Flüssigkeitsfilme an den Gefäßwänden die Messungen stark beeinflussen können. Sind die Medien zudem noch adhesiv mit hoher Viskosität, so ergeben sich weitere Herausforderungen zur korrekten Detektion des Füllstandes. In dieser Arbeit betrachten wir zwei unterschiedliche Technologien, welche eine Mediengefäßerkennung erlauben und die Filmbildung der Medien bei der Füllstandsmessung berücksichtigen.

Technologisch sind zwei Messmethoden für die kapazitive Füllstandmessung weit verbreitet. Im als Charge-TransferMessverfahren bekannten Verfahren wird die Messkapazität, welche stets durch eine geeignete Anordnung von Elektroden zur Anregung des den relevanten Erfassungsbereich abdeckenden elektrischen Feldes besteht, zyklisch Umgeladen [1],[2]. Die dabei erfasste Ladungsmenge $Q$ wird dabei meist auf einer Referenzkapazität integriert und nach einer angemessenen Anzahl von Umladungen erfasst. Durch die Anzahl der Umladungen sowie durch die Größe der Referenzkapazität können die Messparameter des Sensors eingestellt werden. Des Weiteren spielt die Wahl einer geeigneten Umladezeit eine entscheidende Rolle, da die verschiedenen, zu erfassenden Szeanrien mit unterschiedlichen Frequnzgängen aufwarten. Diese Art der Messtechnik wird im Folgenden das erste System für den Performance Vergleich sein.

Ein weiteres, weit verbreitetes Verfahren ist die Einbindung der zu messenden Kapazität in einen resonanten LC-Schwingkreis, in welchem eine Verschiebung der Resonanzfrequenz, je nach Kapazitäts-änderung, getriggert durch z.B. eine Füllstandänderung, stattfindet. Es wird die Verschiebung der Schwingfrequenz messtechnisch erfasst [2]. Dieses Verfahren wird im Folgenden nicht näher betrachtet.

Ein weiterer Ansatz ist durch das innerhlab diesen Papiers dargelegten breitbandigen Messverfahren, welches im Folgenden näher betrachtetwird, gegeben. Gegenüber den bisherigen Messverfahren erfolgt eine Messung mit einem spektral breiten Messsignal. Dies steht im Gegensatz zu den schmalbandigen Messverfahren der zuvor genannten Messmethoden. Dies erlaubt eine bessere Performance des Messsystemes für adhäsive, filmbildende hoch viskose Medien.

\section{Breitbandiges Rauschmessverfahren}

Der Aufbau von kapazitiven Sensoren, welche auf dem Charge-Transfer-Verfahren basieren, ist bekannt und ausreichend in der Literatur dokumentiert [1]. Im Folgenden erfolgt die Vorstellung des zweiten kapazitiven Messverfahren, welches im Weiteren für die viskosen Medien zum Einsatz kommen wird.

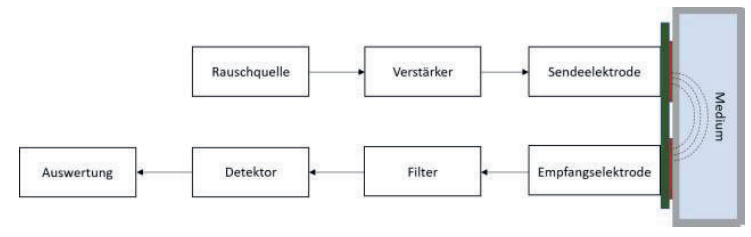

Abb. 2: schematischer Aufbau der breitbandigen Rauschmessung

Der Sensoraufbau erfolgt mittels zwei PCB Elektroden, wobei eine als Sende- und die zweite als Empfangselektrode verwendet wird. Als Anregung wird eine Rauschquelle mit einem breitbandigen Spektrum eingesetzt, welche einen Frequenzbereich im VHF-Band abdeckt. Das erzeugte Rausch-signal wird moduliert, verstärkt und mit einem Anpassnetzwerk auf die Elektrode eingekoppelt. Das Signal an der Empfangselektrode wird nach Filterung auf einen Detektor gegeben und die Leistung gemessen. Im Anschluss erfolgt die Auswertung der Transmission der Messstrecke anhand der Empfangsleistung, wobei durch die eingesetzte Modulation eine hohe Störtoleranz und Genauigkeit erreicht wird.

Gegenüber der im vorherigen Abschnitt erläuterten Messverfahren erfolgt hier eine Anregung in einem breiten Frequenzspektrum anstatt einer Anregungsfrequenz von wenigen $\mathrm{MHz}$ bei dem Charge-Transfer-Verfahren oder einigen $10 \mathrm{MHz}$ bei dem resonanten LCMessverfahren, wodurch eine optimierte Signalantwort seitens des Mediensystems zu erwarten ist.

\section{Präsenzerkennung des Mediengefäßes}

Für eine Präsenzerkennung von Mediengefäßen, wie z.B. bei einem Wassertank, müssen kleine Kapazitätsänderungen detektiert werden. Typische Wandstärken sind z.B. $d_{1}=2-3 \mathrm{~mm}$ für die Gehäusewandung sowie $d_{2}=2-3 \mathrm{~mm}$ für die Mediengefäßwand. Mögliche Materialien sind nicht leitfähige Kunststoffe wie beispielsweise ABS, PP oder PMMA, welche ein $\varepsilon_{r} \approx 1,8-2,4$ aufweisen.

Durch die sehr niedrige Permittivität erfolgt nur eine sehr geringe Kapazitätsänderung $\Delta \mathrm{C}$ im 
Gegensatz zu dem eigentlichen, zu messenden Medium mit $\varepsilon_{\text {Gefäß }} \ll \varepsilon_{\text {Medium. }}$ Um Aussagen über die zu erwartenden Kapazitätsänderungen geben zu können, wurden elektrostatische Feldsimulatonen durchgeführt.

In Abbildung 3 ist eine Feldlinienbild für eine beispielhafte Elektrodenanrodnung speziell zur Erfassung der Präsenz des Mediengefäßes gezeigt. Das gezeigte Szenario beinhaltet zunächst den Sensor und die Montagewand an der der Sensor befestigt wird. Diese Wandung ist in den üblichen Szenarien teil des Gehäuses des jeweiligen Geräts - bspw. einer Kaffeemaschine. Die Feldlinien verlaufen symmetrisch von den anregenden Elektroden hin zur Messelektrode und durchdirngen im wesentlichen die Montagewand, reichen jedoch kaum darüber hinaus.

Fügt man nun das Mediengefäß in die Simulation mit ein (Abbildung 4), so erkennt man eine geringfügige Verformung der Feldlinien einhergehend mit einer geringen messbaren Kapazitätsänderung durch das Mediengefäß.

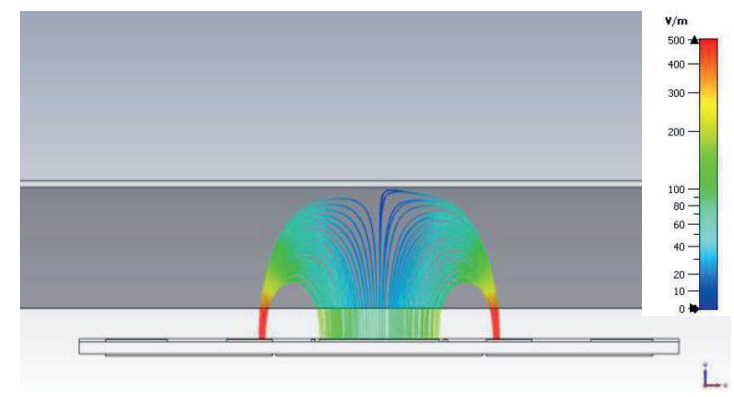

Abb. 3: Feldlinienbild des elektrischen Feldes einer Elektrodenanordnung ohne Mediengefäß, Schnitt durch Sensor$P C B$ (weiß, unten) und Montagewandung (dunkelgrau, mitte) mit Luftbereichen (weiß) und Hintergrundmaterial (Luft, hellgrau, oben)

Betrachtet man die simulierten Kapazitäten bei vorhandenem sowie abwesendem Gefäß in Abbildung 3 und 4, so beträgt die Kapazitätsänderung nur $\Delta \mathrm{C} \approx 10 \mathrm{fF}$. Diese sehr kleine Änderung erfordert weitere Maßnahmen zur Stabilisierung der Messtechnik hinsichtlich Temperatur- und Feuchtigkeitseinflüsse sowie mechanischen Toleranzen der Gesamtanordnung. Eine Verbesserung der Stabilität der Präsenzerkennung des Gefäßes kann durch geeignete, weitere Elektroden und deren gemeinsame Auswertung erreicht werden.

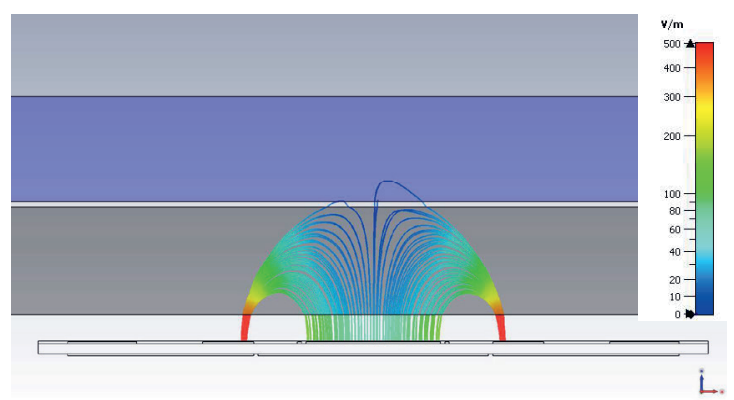

Abb. 4: Feldlinienbild des elektrischen Feldes einer Elektrodenanordnung ohne Mediengefäß, Schnitt durch SensorPCB (weiß, unten), Montagewandung (dunkelgrau, mitte), Gefäßwand (blau, oben) mit Luftbereichen (weiß) und Hintergrundmaterial (Luft, hellgrau, oben)

Die Kompensation der Temperaturabhängigkeit kann weitestgehend elektronisch erfolgen. So ist die Änderung der gemessenen Kapazität im Bereich von $+20^{\circ} \mathrm{C}$ bis $+70^{\circ} \mathrm{C}$ kleiner als $1 \mathrm{fF}$. Der Einfluss der Feuchtigkeit ist dagegen um eine Zehnerpotenz größer als der Einfluss der Temperatur. Deswegen erfolgt eine aktive Korrektur der Kapazitätsmesswerte in der Software, wenn die Umgebungsbedingung der Luftfeuchtigkeit im Bereich von 20-85\% rHd sich befindet.

\section{Modellbildung der Applikationen}

Für die Modellierung der Füllstandmessung wird der vereinfachte schematische Aufbau in Abbildung 5 mit einer Gefäßwand und zwei planaren Elektroden betrachtet.

Als Medium soll hier Wasser im Allgemeinen wie z.B. Leitungswasser betrachtet werden. Wasser weist eine hohe relative Dielektrizitätskonstante von $\varepsilon_{r} \approx 80$ auf. Aufgrund der darin vorhandenen lonen besitzt Leitungswasser ebenfalls eine elektrische Leitfähigkeit, die wesentlich von den darin gelösten Salzen und den weiteren Verunreinigungen bestimmt wird. Abhängig vom Messverfahren beeinflussen kapazitive und resistive Effekte die gemessenen Kapazitätswerte auf unterschiedliche Weise.

In dem in Abbildung 5 dargestellten Ersatzschaltbild modellieren die Kapazitäten $\mathrm{C}_{0}$ die Einkopplung des Signals auf die lokalen Knoten im Sinne von elektrischen Knoten durch die Gefäßwand. Die variable Kapazität $\mathrm{C}_{1}$ stellt den kapazitiven Einfluss der Signalstrecke durch das Medium Wasser dar. 


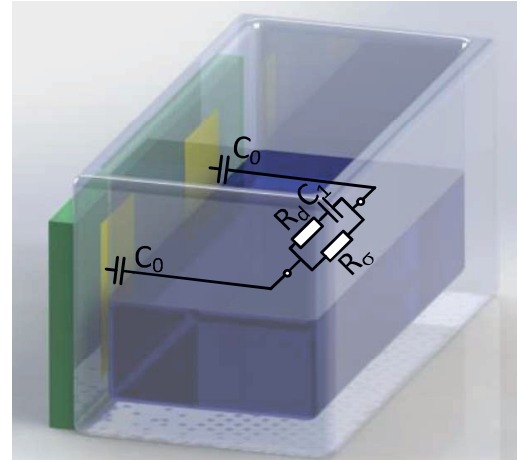

\section{Abb. 5: Elektrotechnisches Ersatzschaltbild für die Modellierung der Applikation.}

Dielektrische Verluste und die Leitfähigkeit des Wassers werden jeweils über die Widerstände $R_{d}$ und $R_{\sigma}$ modelliert.

Bei leitfähigen, ionenreichen Flüssigkeiten ist bereits bei einem sehr dünnen Flüssigkeitsfilm auf der Tankwand der Widerstand $R_{\sigma}$ in Abhängigkeit der verwendten Messfrequenz vergleichsweise klein. In diesem Fall kann mit einer einfachen Messung nicht mehr unterschieden werden ob der Erfassungsbereich des Sensors großflächig mit einem Wandfilm überzogen ist oder der Füllstand tatsächlich ein hohes Level erreicht hat, da die Größe $C_{1}$, welche zu einem erheblichen Teil den Informationsgehalt über den tatsächlichen Füllstand trägt, nicht mehr gemessen werden kann. Um dieses Verhalten zu untersuchen werden Messungen an einem Referenzaufbau durchgeführt. Dabei werden leitfähige Filme unterschiedlicher Dicke durch in isotonische Kochsalzlöung (9g NaCl pro 1l Lösung) getränkte Papierstreifen nachgestellt.

\section{Messung mit dem Charge-Transfer Verfahren}

Die Messung erfolgt zunächst mithilfe eines Sensors der nach dem Charge-TransferVerfahren mit einstellbarer Umladezeit arbeitet. Eine $3 \mathrm{~mm}$ dicke PMMA-Platte bildet die Tankwand zwischen Sensor und Papierstreifen nach.

Durch Messungen mit unterschiedlichen Umladezeiten kann der Einfluss des Widerstand $R_{\sigma}$ auf die Kapazitätsmessung anhand der RC-Zeitkonstante des Gesamtsystems untersucht werden. Hierbei ist es wichtig zu verstehen, dass die relevante Zeitkonstante nicht allein durch die Messkapazität nach Abbildung 5 bestimmt ist, sondern zusätzlich beschränkende Elemente in der Messchaltung mitwirken.
Die gemessene Abweichung der Kapazität durch die Präsenz von einem oder mehreren $100 \mu \mathrm{m}$ starken Papierstreifen gegenüber dem Messwert ohne Papierstreifen ist in Abbildung 6 für Umladezeiten von 21ns bis 188ns dargestellt. Erkennbar ist die durch die Leitfähigkeit des durch das Papier gebundenen ionischen Films bestimmte Ladekurve der resultierenden RC-Schaltung, welche direkt die gemessene Ladungsmenge bestimmt. Bei dickeren Schichten ab etwa 4 Papierstreifen bestimmt zunehmend nicht mehr der Filmwiderstand, sondern die Komponenten der Schaltung die Zeitkonstanten.

Zeitkonstanten im Nanosekundenbereich stellen Sensoren, die nach dem ChargeTransfer-Verfahren arbeiten, vor Herausforderungen. Dies gilt insbesondere für Sensoranwendungen welche einen hohen Fokus auf die Herstellkosten aufweisen und auf diskrete Bauteile basieren. Die mimimale Umladedauer ist oft durch die Taktfrequenz des ansteuernden Mikrocontrollers begrenzt. Die verwendeten Analogschalter müssen entsprechend schnell schalten, ebenfalls muss das Timing für sämtliche relevanten Schaltungsteile über alle Betriebbedingungen sichergestellt werden. Eine weitere Limitierung besteht in der begrenzten Treiberfähigkeit der I/O-Pins gängiger Mikrocontroller in Verbindung mit großflächigen Messelektroden. Typischerweise müssen bei dieser Art Sensor Kapazitäten im Picofaradbereich umgeladen werden. Zusätzlich wirken parasitäre Kapazitäten im Bereich von einigen zehn Picofarad.

Anhand von Messungen mit mehreren Umladezeiten kann, für bekannte Flüssigkeiten, eine dünne Flüssigkeitsschicht erkannt, sowie die Dicke der Schicht abgeschätzt werden. Der betrachtete Sensor ist in der Lage die typischerweise verbleibenden Flüssigkeitsfilme bei der Füllstandmessung von Wasser zu erkennen sowie deren Einfluss zu kompensieren. Ebenfalls ist eine Präsenzdetektion des Tanks sowie eine kontinuierliche Füllstandmessung möglich. Mit zusätzlichen Techniken können bei dieser Art der Messung Einflüsse durch Berührung der Flüssigkeit sowie durch parasitäre Kapazitäten zur Sensorumgebung minimiert werden. 


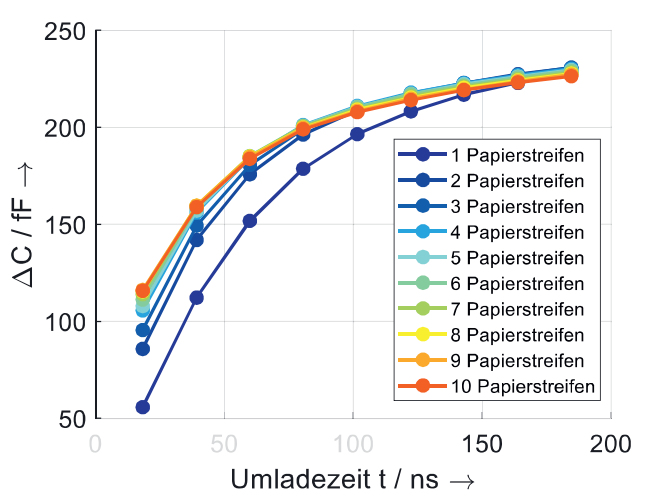

Abb. 6: Kapazitätsänderung von Filmen mit erhöhter Leitfähigkeit, hier $\mathrm{NaCl}$.

\section{Messung mit breitbandigem Rauschmess- verfahren}

Die Füllstandmessung von stark leitfähigen, zähflüssigen Medien mit dicken, verbleibenden Flüssigkeitsfilmen stellt die Messtechnik aufrgund der daraus resultierenden sehr kurzen Zeitkonstanten nach Abbildung 5 vor besondere Herausforderungen. In diesem Fall muss angestrebt werden trotz der hohen Leitfähigkeit $R_{\sigma}$ tatsächlich die Kapazität $C_{1}$ auszuwerten. Der Füllstandsensor arbeitet deshalb mit Signalfrequenzen im Bereich von einigen bis zu mehreren hundert $\mathrm{MHz}$. Dabei wird ein breitbandiges, weißes Rauschsignal moduliert und die von der Sendeelektrode auf die Empfangselektrode gekoppelte Leistung des Nutzsignals gemessen. Somit ist der Sensor in der Lage, Füllstände auch unter erschwerten Bedingungen, wie beispielsweise bei Einsatz von Waschmitteln oder Milch, zuverlässig zu bestimmen.

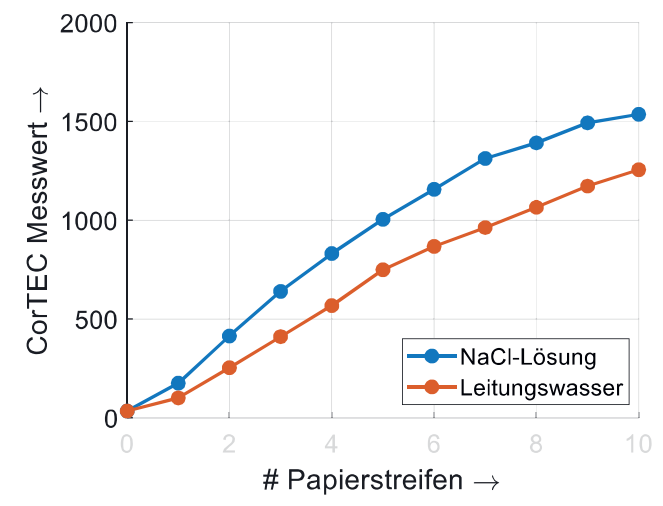

Abb. 7: Betragsänderung bei variierender, simulierter Filmdicke leitfähiger Medien.

Abbildung 7 zeigt den Verlauf des Messwertes des Sensors für ein bis zehn Lagen in Leitungswasser sowie in Kochsalzlösung getränkte Papierstreifen. Untersuchungen haben gezeigt, dass getränkte Papierstreifen ein repräsentatives Verfahren darstellt um Filme zu simulieren. Der Messaufbau entspricht dem des auf dem Charge-TransferVarfahren basierendem Vergleichssensor. $\mathrm{Da}$ bei dieser Messung im Wesentlichen der kapazitive Teil $\mathrm{C}_{1}$ der Messstrecke ausgewertet wird, kann der Sensor selbst noch bspw. die neunte von der zehnten Papierschicht unterscheiden. Vergleichbare Ergebnisse liefert der Sensor ebenfalls für Papierstreifen, die sowohl in NaCl-Lösung als auch in destilliertem Wasser getränkt werden.

\section{Ergebnis}

Die Diskussion der Ergebnisse zeigt, dass das auf dem Charge - Transfer basierenden Messverfahren für wässrige Medien mit dünnen Wandfilmen sinnvoll einsetzbar ist. Für hoch viskose Medien jedoch erweist sich der Einsatz eines Messverfahrens mit höherer Messfrequenz, wie z.B. das in dieser Untersuchung eingesetzte modulierte breitbandige Rauschsignalverfahren, als vorteilhaft, da hier die hohe Leitfähigkeit der Wandfilme geringere Einflüsse auf das Messergebnis haben und die tatsächliche Kapazität sowie deren Änderung erfasst wird.

Beide Verfahren sind in der Lage, die geringe Kapazitätsänderung für die Präsenzerkennung des Mediengefäßes reproduzierbar zu erkennen und den Füllstand von wässrigen Medien sowie von adhäsiven, viskosen Medien trotz Filmbildung zu erfassen.

\section{Literaturnachweis}

[1] P. Hal, Sensor Review 19, Issue 2, 96-105 (1999)

[2] W. Du, Series in Sensors, CRC Press, (2015) 